\title{
Planetesimal Formation by Gravitational Instability
}

\author{
Andrew N. Youdin ${ }^{1}$ \\ Department of Physics, 366 Leconte Hall, University of California, Berkeley, CA 94720 \\ Frank H. Shu ${ }^{1}$ \\ Department of Physics, National Tsing Hua University, 101, Sec. 2, Kuang Fu Rd., \\ Hsinchu 30013, Taiwan, R. O. C. \\ youd, shu@astron. berkeley.edu
}

\begin{abstract}
We investigate the formation of planetesimals via the gravitational instability of solids that have settled to the midplane of a circumstellar disk. Vertical shear between the gas and a subdisk of solids induces turbulent mixing which inhibits gravitational instability. Working in the limit of small, well-coupled particles, we find that the mixing becomes ineffective when the surface density ratio of solids to gas exceeds a critical value. Solids in excess of this precipitation limit can undergo midplane gravitational instability and form planetesimals. However, this saturation effect typically requires increasing the local ratio of solid to gaseous surface density by factors of two to ten times cosmic abundances, depending on the exact properties of the gas disk. We discuss existing astrophysical mechanisms for augmenting the ratio of solids to gas in protoplanetary disks by such factors, and investigate a particular process that depends on the radial variations of orbital drift speeds induced by gas drag. This mechanism can concentrate millimeter sized chondrules to the supercritical surface density in $\leq$ few $\times 10^{6}$ years, a suggestive timescale for the disappearance of dusty disks in $\mathrm{T}$ Tauri stars. We discuss the relevance of our results to some outstanding puzzles in planet formation theory: the size of the observed solar system, and the rapid type I migration of Earth mass bodies.
\end{abstract}

Subject headings: planetary systems: formation — planetary systems: protoplanetary disks — instabilities — turbulence

\footnotetext{
${ }^{1}$ Also: Department of Astronomy, 601 Campbell Hall, University of California, Berkeley, CA 94720
} 


\section{Introduction}

The planetesimal hypothesis states that terrestrial planets and the icy cores of gas giants formed in a disk by the accretion of smaller solid bodies, called planetesimals. Planetesimals are defined as primitive solids of kilometer size or larger. Such a scale is meaningful because it delimits where pairwise gravity dominates gas drag, leading to the runaway or oligarchic growth of protoplanets (Lissauer 1993). Because planetesimal formation must occur, at least for the giant planets, while gas is still present in the solar nebula, the coupling of gas and solids may be crucial to understanding the process.

The prevailing view for planetesimal formation posits agglomerative growth from submicron-sized "interstellar" grains to km-sized bodies (cf. the review of Lissauer 1993). However, building planetesimals through pure solid-state sticking forces has many problems.

First, in the inner solar system, interior to the "snow line," the only solids available for the formation of the terrestrial planets were rocks. Everyday experience tells us that dry silicate particulates of millimeter and larger size, such as sand, do not stick at almost any speed of attempted assemblage. Measurements of interparticle collisions in microgravity experiments reinforce these intuitive impressions; indeed, $\mu \mathrm{m}$ sized dust particles disrupt larger aggregates upon collision at relative velocities $>\mathrm{m} / \mathrm{s}$ (Blum \& Wurm 2000). For collisions between fluffy mm-sized agglomerations of $\mu \mathrm{m}$ sized grains, disruption also occured at speeds $>\mathrm{m} / \mathrm{s}$. However at lower speeds, down to $15 \mathrm{~cm} / \mathrm{s}$, only restitution (bouncing) was observed, without any sticking (Blum \& Muench 1993). Relative velocities considerably higher than $1 \mathrm{~m} / \mathrm{s}$ arise from chaotic motions in a turbulent disk or from differential orbital drift in a laminar disk (Weidenschilling \& Cuzzi 1993). For mm-sized crystalline silicates, such as chondrules (see below), significant shattering occurs only at collision speeds $>$ several $\mathrm{km} / \mathrm{s}$ (Jones, Tielens, \& Hollenbach 1996). Thus, compact pieces of rock that make up the bulk of the material of chondritic meteorites, whose parent bodies are primitive asteroids (rocky planetesimals), are unlikely either to agglomerate or to fragment at the collisional velocities common in the nebular disk when there is still appreciable gas present to exert appreciable drag on the solids (the well-coupled limit, see Appendix).

Second, at temperatures significantly below their melting points, even ices in the outer solar system may not be much more sticky. Experiments by Supulver et al. (1997) found that water frost has spring-like properties and can only induce sticking for collision speeds $<0.5 \mathrm{~cm} / \mathrm{s}$. Furthermore, if ice balls could grow by continued agglomeration until disruptive tidal forces became stronger than the cohesive strength of crystalline ice, we should expect many icy particulates in Saturn's rings to acquire sizes of order $\sim 10-100 \mathrm{~km}$. In fact, except for the occasional embedded moonlet (whose origin may lie in the fragmentation of yet larger bodies rather than from the assemblage of ordinary ring particles), the particulates 
in Saturn's rings have a maximum size $\sim 5 \mathrm{~m}$ (Zebker, Marouf, \& Tyler 1985), intriguingly close to the value implied if collective self-gravity were the only available force to assemble the largest bodies (Shu 1984).

Third, if we examine the most primitive meteorites, the ordinary and carbonaceous chondrites, we find no evidence for a continuous range of particulates spanning a range of sizes from less than a micron to, say, a meter or more (say, comparable to the size of the entire meteorite). Instead, we find the largest mass fraction of such objects to be composed of chondrules: quasi-spherical, once molten, inclusions of millimeter and smaller sizes that give evidence of once having been intensely and briefly heated (perhaps multiple times). This fact suggests that sticky agglomerative events, such as those experienced by compound chondrules (Rubin 2000), required special transient heating events to overcome the obstacle that liquids are not stable thermodynamic phases for any temperature at the low pressures prevalent in protoplanetary disks. In any case, from the meteoritic record, the early solar system failed to generate by primitive processes any compact particulates in excess of a few centimeters in size (the largest refractory inclusions). (See Shu et al. (2001) for a promising, although unconventional, mechanism for producing the refractory inclusions and chondrules in chondritic meteorites.) Electrostatic attraction could have played a role in building looser aggregates if the individual particulates acquired significant levels of charge (Marshall \& Cuzzi 2001). This effect has been seen in zero-gravity experiments with particle densities well above the threshold for gravitational instability. In order to be a relevant growth mechanism, it must be shown that tribocharging, the balance between collisional charging and ion/electron discharging, yields electrostatic attraction at much lower particle densities.

Fourth, even if a mechanism could be found to grow chondrule-sized particulates to meter-sized bodies, one would have to worry about the rapid inward orbital drift associated with gas drag that would carry such bodies from 1 AU into the protosun on a time scale of only $\sim 10^{2}$ yr (Weidenschilling 1977). In contrast, mm- and km-sized bodies have gas-drag drift times in excess of $10^{5} \mathrm{yr}$. Only by the direct assemblage of chondrules and related objects into planetesimals, avoiding intermediate steps, can one prevent a rapid loss of solid material from the solar nebula by gas drag.

Such a direct-assemblage mechanism exists in the gravitational instability (GI) proposal put forth by Goldreich \& Ward (1973). A similar theory was advanced independently by Safronov (1969). In the Goldreich-Ward theory, particulate settling yields a subdisk of solids that is thin and non-dispersive enough to make overdense regions undergo runaway local contraction. This occurs when Toomre's criterion for axisymmetric GI in a rotating 
disk (the nonaxisymmetric case is similar) is satisfied:

$$
Q_{\mathrm{p}} \equiv \frac{\Omega c_{\mathrm{p}}}{\pi G \Sigma_{\mathrm{p}}}<1
$$

where $\Omega$ is the angular Keplerian rotation rate, and $c$ and $\Sigma$ are the velocity dispersion and surface density. Throughout we use "p" and "g" subscripts to refer, respectively, to the particle and gas components of the disk. The Goldreich-Ward instability should not be confused with the mechanism of Boss (2000), who considers the formation of coreless gas giant planets from GI of gas disks.

Toomre's criterion (1) is equivalent (within factors of order unity) to the "Roche" limit which has been derived specifically for the case of stratified fluids(Goldreich \& Lynden-Bell 1965; Sekiya 1983). Sekiya finds GI to occur when the particulate plus gas space-density, $\rho=\rho_{\mathrm{g}}+\rho_{\mathrm{p}}$, at a distance $r$ from a star of mass $M_{*}$ exceeds a certain critical value in the midplane:

$$
\rho>\rho_{\mathrm{R}} \equiv 0.62 M_{\star} / r^{3} \text {. }
$$

At a distance $r=1$ AU from the Sun, $\rho_{\mathrm{R}}=4 \times 10^{-7} \mathrm{~g} / \mathrm{cm}^{3}$, which implies a critical space density of rock that is roughly seven orders of magnitude less than the internal density of compact rock. Thus, in the stages preceding actual planetesimal formation, we may treat the collection of solids as an additional ideal "gas" co-mixed with the real gas of the system.

Operating at a radius of $r=1 \mathrm{AU}$, the self-gravitating disturbance with the most unstable wavelength creates $\sim 5 \mathrm{~km}$ planetesimals in $\sim 10^{3} \mathrm{yr}$ (Youdin \& Shu 2002). The process occurs on a time scale longer than orbital periods $(\sim 1 \mathrm{yr}$ in the zone for terrestrial planet formation) because of the need to damp spin-up and random velocities during the contraction to planetesimal densities. But the important point remains, that by leapfrogging intermediate size regimes, GI avoids the rapid inspiral of meter-sized bodies.

Unfortunately, a powerful argument has been developed against the GI scenario, which has led largely to its abandonment by modern workers in the field (Weidenschilling 1995). A review of the difficulty is necessary before before we can justify a renewed attack on the basic idea.

Even in an otherwise quiescent disk, midplane turbulence may develop to stir the particulate layer too vigorously to allow sufficient solid settling to the midplane. Without such settling the criterion (2) cannot be satisfied. The problem lies in the vertical shear possessed by disks with a highly stratified vertical distribution of solid to gas. The particulatedominated sub-disk, which possesses near-Keplerian rotation, revolves somewhat faster than the surrounding gas disk, which has non-vanishing support against the inward pull of the Sun from gas pressure in addition to centrifugal effects. The magnitude of the resulting 
velocity differential, $\Delta v_{\phi}=\eta v_{\mathrm{K}}=\eta r \Omega$, is proportional to $\eta$, which roughly equals the ratio of thermal to kinetic energy of the gas:

$$
\eta \equiv-\frac{(\partial P / \partial r)}{\left(2 \rho_{\mathrm{g}} r \Omega^{2}\right)} \sim\left(c_{\mathrm{g}} / v_{\mathrm{K}}\right)^{2},
$$

where $P$ is the gas pressure and $c_{\mathrm{g}}$ is the isothermal sound speed.

In the popular model of the minimum solar nebula (hereafter MSN, see $\S 2$ ), $\eta \simeq 2 \times$ $10^{-3}(r / \mathrm{AU})^{1 / 2}$, and $\Delta v_{\phi} \simeq 50 \mathrm{~m} / \mathrm{s}$ at $r=1 \mathrm{AU}$ (Hayashi 1981). Turbulent eddies with a characteristic velocity equal to the available velocity differential, $\sim \Delta v_{\phi}$, would then prevent GI, since the Toomre $Q$ criterion requires that the particle random velocity be much smaller: $c_{\mathrm{p}}<7 \mathrm{~cm} / \mathrm{s} \ll \Delta v_{\phi}$, for instability (Weidenschilling 1995).

These general arguments are supported by numerical simulations (Cuzzi, Dobrovolskis, \& Champney 1993) which calculate the steady state properties of two-phase (gas and particulate) turbulence in the midplane of a MSN disk. Particulates with internal densities of normal rock and with sizes from 10 to $60 \mathrm{~cm}$ (which are assumed to have grown by other mechanisms and are moderately coupled to gas motions) acquire space densities too low for GI by an order of magnitude or more. Their computational methods use a mixing length prescription to relate diffusivity and velocity shear through an extrapolation from laboratory studies of boundary layers. While quite sophisticated, this approach does not directly address the mechanism by which the existence of the vertical shear generates turbulence.

Using a linear stability analysis, Sekiya (1998) confirmed that GI in a turbulent dust layer is impossible, unless the ratio of dust to gas surface densities is significantly enhanced over normal cosmic values. Part of the purpose of the current paper is to understand the physical basis of Sekiya's conclusion. But for the present, let us merely note that there exist several possibilities for enhancing the solid/gas ratio in protoplanetary disks, either globally or by local concentration.

First, in the X-wind model (Shu, Shang, \& Lee 1996), chondrules are created from material at the magnetically truncated inner edge of the disk, called the X-point. Solids and gas are launched from the X-point in a bipolar outflow. While the gas escapes in a collimated jet, solids of roughly millimeter size fall back to the disk at planetary distances, thus increasing the disk's solid/gas ratio. Since $1 / 3$ of all material which passes through the $\mathrm{X}$-point is launched in an outflow, while the remaining $2 / 3$ accretes onto the protostar, rocky material in the disk could be augmented by a total amount as much as $4 \times 10^{30} \mathrm{~g}$, or 30 times the amount of rock in a standard MSN model. Such an enhancement factor is more than sufficient, as we shall see, to promote GI in the sub-disk of solids. In point of fact, because of efficiency considerations in the manufacture of chondrules and refractory inclusions and 
their irradiation to produce short-lived radionuclides (see, e.g., Gounelle et al. 2001), there are reasons to believe that an amount of rock not much larger (but perhaps a few times larger) than that contained in a MSN was recycled by the X-wind to the disk. We shall find that what is important is the ratio of solid to gas surface densities in the disk. Once we accept that this ratio need not be cosmic (e.g., rock to gas $=4 \times 10^{-3}$ ), then there exists no a priori theoretical objection to a revival of the Goldreich-Ward mechanism for forming planetesimals.

Second, since solids tend to settle towards the midplane, the surface layers of a disk should become relatively gas-rich. Thus, any mechanism that removes material from the surface of a stratified disk would increase the solid to gas ratio computed in terms of vertically projected column densities. Possibilities for such surface removal include (1) photoevaporation, which dominates in the loosely bound outer disk (Shu, Johnstone, \& Hollenbach 1993); (2) layered accretion, which occurs if only the surface layers of a disk are sufficiently ionized to support magneto-rotational turbulence (Gammie 1996); and (3) stripping by stellar winds, which is probably more effective near than far from the star (Hollenbach, Yorke, \& Johnstone 2000). The amounts of solid to gas enhancements achievable by these processes are difficult to predict, but the timescales for the dominant processes are typically $<$ few $\times 10^{6}$ years, and thus likely to be relevant to the evolution of protoplanetary disks.

Third, gas drag can also lead to local enhancement of particulate concentrations by a variety of mechanisms. (1) Isotropic turbulence with a Kolmogorov spectrum concentrates particles in numerical (Squires \& Eaton 1991) and laboratory (Fessler, Kulick, \& Eaton 1994) experiments. Extrapolation to the high Reynolds numbers of protoplanetary disks implies concentration of chondrules by factors of up to $10^{5}$ (Cuzzi et al. 2001). However, these estimates do not take into account the redispersal of the concentrated pockets of solids if the turbulent eddies are intermittent and do not maintain fixed centers. (2) Similarly, disk vortices could concentrate chondrules as well, but they are more effective for meter sized bodies (de la Fuente Marcos \& Barge 2001). There also remains the issue whether vortices will rise spontaneously in protoplanetary disks if there are no natural stirring mechanisms. (3) Secular instabilities associated with gas drag might concentrate particulates, even without self-gravity (Goodman \& Pindor 2000). Goodman and Pindor assumed that gas drag acts collectively on a particulate "sheet", a valid approximation when turbulent wakes overlap. Unfortunately, wake overlap seems unlikely unless the particles are fairly closely packed, in which case GI would already be effective. In a related context, Ward (1976) has shown in an underappreciated study that viscous drag modifies the standard GI criterion through the introduction of an additional instability that can occur at values of $Q \gg 1$. However, this additional instability, being secular in nature, has a much smaller growth rate than the usual Goldreich-Ward mechanism. (4) In $\S 4$, we develop the simplest concentration mechanism 
for particulates: radial migration due to gas drag.

The fundamental assumptions required for the results of this paper are the existence, at some epoch of the disk's evolution, of (1) relatively quiescence in the midplane regions, even though the surface layers may be undergoing active accretion (Gammie 1996), and (2) compact solids with chondrule-like properties that are well, but not perfectly, coupled to the gas through mutual drag.

Under these conditions, we argue (1) that vertical shear can only induce a level of midplane turbulence that has limited ability to stir solids, with the critical value of the surface density of solids being given roughly by $\Sigma_{\mathrm{p}, \mathrm{c}} \sim \eta r \rho_{\mathrm{g}}$; and (2) that gas drag alone can lead to a global radial redistribution of solids so that the local surface density of solids, $\Sigma_{\mathrm{p}}$, can exceed the critical value, $\Sigma_{\mathrm{p}, \mathrm{c}}$. Therefore, whether a recycling of solids occurs by the X-wind mechanism or a radial redistribution of solids occurs by simple gas drag, we conclude that the planet-forming zones of the primitive solar system can achieve the requisite conditions for the formation of planetesimals on a time scale comparable to the typical lifetime, $\sim 3 \times 10^{6} \mathrm{yr}$, that has been inferred for the disks of T Tauri stars (Haisch, Lada, \& Lada 2001). However, the margin for success is not large, and it could be that planet formation is a less universal phenomenon than it has been widely touted to be, and that it has a much greater diversity of outcomes (including a complete failure to form any planets) than suspected prior to the discovery of extrasolar planets (Mayor and Queloz 1996, Marcy and Butler 1998). Indeed, the very fact that planetesimal formation may involve a threshold phenomenon, namely the existence of a nontrivial critical surface density, $\Sigma_{\mathrm{p}, \mathrm{c}} \sim \eta r \rho_{\mathrm{g}}$, implies that low-metallicity systems should be much less likely to form planets than high-metallicity systems, a correlation which seems already to be present in the empirical literature (Gilliland et al. 2000, Laughlin 2000).

This paper is organized as follows. In $\S 2$ we present the basic properties of our disk models. After reviewing the techniques of Sekiya (1998) for deriving density distributions of well-coupled particles in $\S 3.1$, we physically interpret, in $\S 3.2$, the midplane density singularities which occur in these profiles as evidence that midplane turbulence can only stir a finite amount of material. This allows us, in $\S 3.3$ to calculate the solid/gas enhancements required for GI in various model disks. We show that aerodynamic drift can concentrate particles of a given size radially in the disk on cosmogonically interesting time scales in $\S 4.1$, and we generalize to distributions of particle sizes in $\S 4.2$. In $\S 4.3$ we evaluate whether this concentration mechanism provides enough enhancement to induce GI. Closing remarks are made in $\S 5$. 


\section{Disk Properties}

If any accretion and its associated turbulence occurs in the disk, we assume that they are confined to the top and bottom surface layers of the disk (Gammie 1996). Because the deeper (midplane) layers of the disk are then heated only by radiation from above or below, we can then model the gaseous component as being in hydrostatic equilibrium with a vertically isothermal distribution of temperature, while the radial distributions for the temperature and surface density are taken, for simplicity, to have simple power-law profiles:

$$
\begin{aligned}
T & =280 f_{T} \varpi^{-q} \mathrm{~K}, \\
\Sigma_{g} & =1700 f_{g} \varpi^{-p} \mathrm{~g} \mathrm{~cm}^{-2} .
\end{aligned}
$$

The normalization occurs relative to $1 \mathrm{AU}$; i.e., we define a dimensionless radius, $\varpi \equiv r / \mathrm{AU}$, and then $280 f_{T}$ and $1700 f_{g}$ represent the gas temperature (in K) and surface density (in $\mathrm{g} / \mathrm{cm}^{2}$ ) at $1 \mathrm{AU}$. The power-law indices, $q$ and $p$, are the remaining parameters that define our gas disk. In the MSN model advocated by (Hayashi 1981), $f_{T}=f_{g}=1, p=3 / 2$, and $q=1 / 2$; but for completeness, we shall consider broader ranges of possible models.

We ignore details of the vertical structure of the gas disk, because our interest lies in the midplane region, to which particles settle on a timescale (Goldreich \& Ward 1973):

$$
t_{\text {set }} \sim \frac{\Sigma_{\mathrm{g}}}{\rho_{s} a \Omega} \sim 10^{6}\left(\frac{\mu \mathrm{m}}{a}\right) \mathrm{yr} .
$$

In the above, $a$ is the particle radius and $\rho_{\mathrm{s}}$ is the internal density of the solid material, typically $3 \mathrm{~g} / \mathrm{cm}^{-3}$ for rock and $1 \mathrm{~g} / \mathrm{cm}^{-3}$ for ice.

We shall initially consider rock (r) and ice (i) distributions that reflect the power-law distributions of the gas:

$$
\begin{aligned}
& \Sigma_{\mathrm{r}}=7.1 f_{\mathrm{r}} \varpi^{-p} \mathrm{~g} \mathrm{~cm}^{-2}, \\
& \Sigma_{\mathrm{i}}=\left\{\begin{array}{cll}
0 & \text { if } T(\varpi)>170 \mathrm{~K} \\
23 f_{\mathrm{i}} \varpi^{-p} \mathrm{~g} \mathrm{~cm}^{-2} & \text { if } & T(\varpi)<170 \mathrm{~K}
\end{array},\right.
\end{aligned}
$$

where the total surface density of solids is: $\Sigma_{\mathrm{p}}=\Sigma_{\mathrm{r}}+\Sigma_{\mathrm{i}}$. At cosmic proportions, $f_{\mathrm{g}}, f_{\mathrm{r}}$, and $f_{\mathrm{i}}$ would all be equal to each other (and equal to 1 in the Hayashi model), but we shall relax this restrictive assumption in what follows.

Table 1 shows the parameters for the MSN and other models used in this paper. Values of the reference state at solar abundances are shown. The first letter of a model: H, A, or B; indicates its temperature profile: warm (Hayashi's MSN values), cool, or cold; respectively. The surface densities are consistent with the MSN $\left(p=3 / 2, f_{\mathrm{r}}=1\right)$ unless denoted otherwise, e.g. appending an " $\mathrm{f}$ " to indicate a flatter $p=1$ profile. 
Our temperature and surface density profiles are chosen to lie within the bounds set by astronomical observations, particularly the mm-wave continuum emission from $\mathrm{T}$ Tauri disks (Osterloh \& Beckwith 1995), as well as with the midplane temperatures predicted by the theory of passive, flared disks (Chiang \& Goldreich 1997). These considerations yield icelines considerably interior to Jupiter's 5.2 AU orbit. Uncertainties in opacities mean that while the particle mass in most $\mathrm{T}$ Tauri disks is at least that of the MSN, it could be larger if mass is hidden in particulates much larger than the wavelength of the observations. While ISO detected warm $\mathrm{H}_{2}$ around T Tauri stars (Thi et al. 2000), more detections with higher singal-to-noise are needed to give stringent constraints on gas mass.

\section{The Connection Between Solid/Gas Ratio and Gravitational Instability}

\subsection{Dust Density Profiles}

We briefly review Sekiya's (1998) technique for deriving dust density profiles before interpreting the singular cusps which appear in these profiles. For small particles which are well-coupled to gas motions, i.e. when the particle stopping time is shorter than the dynamical and eddy turnover times, the gas-solid mixture can be thought of as a single stratified fluid. This limit applies very well to mm-sized and smaller particles, however the particles must be large enough so that their settling times, see (6), are shorter than the time since global turbulence becomes weak enough to allow particulate settling. The well-coupled limit is the most conservative assumption in which to demonstrate GI since larger, decoupled solids are stirred less efficiently by gas turbulence.

Radial hydrostatic balance yields an orbital velocity of the combined fluid which depends on the vertical distribution or particles (Nakagawa, Sekiya, \& Hayashi 1986):

$$
v_{\phi}(z)=\left[1-\eta \frac{\rho_{\mathrm{g}}}{\rho_{\mathrm{p}}(z)+\rho_{g}}\right] v_{\mathrm{K}}, .
$$

To derive the above, we have ignored the variation of the gas density in the vertical direction since the particulate subdisk is much thinner than a gas scale height. When solids provide most of the inertia, $\rho_{\mathrm{p}} \gg \rho_{\mathrm{g}}$, the fluid motion is Keplerian, but when gas dominates we have the usual formula for pressure-supported rotation, $v_{\phi}=(1-\eta) v_{\mathrm{K}}$.

As particles settle towards the midplane, the vertical shear rate (which promotes mixing) and the buoyancy (which stabilizes against mixing) both increase. This competition between destabilizing and stabilizing influences is conventionally characterized by the Richardson 
number:

$$
R i=\frac{N^{2}}{\left(\partial v_{\phi} / \partial z\right)^{2}}
$$

In the above formulation, the Brunt-Vaisala frequency, $N$, is a measure of buoyancy:

$$
N^{2} \equiv g_{z} \partial \ln \rho / \partial z
$$

where $g_{z}$ is the vertical gravity. When the $R i$ drops below a critical value, $R i_{\mathrm{c}}$, typically $\approx 1 / 4$, we have Kelvin-Helmholtz instability (KHI) and the onset of midplane turbulence.

At low particulate densities $\rho_{\mathrm{p}} \ll \rho_{\mathrm{g}}$, we may approximate the vertical gravity as coming solely from the central star, $g_{z} \approx \Omega^{2} z$, while differentiation of equation (9) yields for equation (10):

$$
R i \propto \frac{\rho_{\mathrm{g}} z}{\partial \rho_{\mathrm{p}} / \partial z}
$$

Increasing stratification is then destabilizing, since the increase in shear outweighs the increasing buoyancy, decreasing $R i$ until it reaches $R i_{\mathrm{c}}$.

In our detailed calculations, we include the self-gravity of the combined fluid in the plane-parallel approximation:

$$
g_{z}=\Omega^{2} z+4 \pi G \int_{0}^{z}\left[\rho_{\mathrm{g}}+\rho_{\mathrm{p}}\left(z^{\prime}\right)\right] d z^{\prime} .
$$

When $\rho_{\mathrm{p}}$ becomes large because of the settling of particulates to the midplane, the self-gravity of sub-disk can provide a significant stabilizing influence against KHI. Indeed, we shall find that this additional effect is the cause of a sudden "precipitation phenomenon" when the surface density of solids crosses a critical value. Since the self-gravity of the subdisk of solids is also of crucial importance for the Goldreich-Ward mechanism, we find that "precipitation" is virtually synonymous with the satisfaction of the criterion for GI, equation (2).

To see this, note that for the second term on the right-hand side of equation (13) to become of comparable importance as the first, we require

$$
4 \pi\left(\rho_{\mathrm{g}}+\rho_{\mathrm{p}}\right) \sim \Omega^{2} / G=M_{\star} / r^{3},
$$

which differs from equation (2) only by a factor of 7.8. Precipitation is formally reached before GI (if we think of a process of slowly increasing the local solid/gas ratio in the disk), but since precipitation results, as we shall see, in the settling out of a very dense midplanelayer of solids, GI follows almost immediately after conditions in the disk become ripe for the beginning of precipitation.

Before these dramatic events occur, the particulate layer should reach an equilibrium which is marginally unstable to the KHI, because even weak turbulence is sufficient to halt 
the settling of well-coupled particles. We henceforth assume with Sekiya that the subdisk of solids satisfies at each value of $z$, the marginally stable condition, $R i=R i_{\mathrm{c}}=1 / 4$, consistent with the necessary criterion for instability in plane-parallel flows. The result is probably modified by the disk geometry and Coriolis forces. However, preliminary investigations of an analogous two-layer model (Youdin, unpublished) indicate that instability is not significantly altered by the inclusion of rotation, though the analog of $R i_{\mathrm{c}}$ decreases slightly. In any event, meaningful quantities (particle scale height and critical surface density) scale as $\sqrt{R i_{\mathrm{c}}}$, so the exact value used for the critical Richardson number does not have a strong effect on the numerical results.

The ansatz of a marginally stable state, $R i=1 / 4$, defines via (10) and (13) an integrodifferential equation for particle density profiles, $\rho_{\mathrm{p}}(z)$, at a given radius. The solution [see eqn. (18) in Sekiya (1998)], requires two boundary conditions: the midplane particulate density, $\rho_{\mathrm{p}}(0)$, and reflection symmetry across the midplane, $\partial \rho_{\mathrm{p}} / \partial z=0$. The former is equivalent, as a one-to-one mapping, to an integral constraint on $\Sigma_{\mathrm{p}}$.

Density profiles of this type can be analyzed for GI, e.g. using (2). One typically finds that low mass disks are stable to the Goldreich-Ward mechanism at cosmic abundances for solids/gas. However increasing the surface density in particles, $\Sigma_{\mathrm{p}}$, while holding $\Sigma_{\mathrm{g}}$ fixed, leads to the development of a density cusp (see Fig. 1), which quickly leads to GI in the midplane, as we described above. Decreasing $\Sigma_{\mathrm{g}}$ relative to $\Sigma_{\mathrm{p}}$ has the same effect. In this context, it is well to recall that the evidence of the planets of the solar system tells us only what the minimum content of the solids was in the primitive solar nebula; it says almost nothing about either the maximum content of solids or actual content of gas at the time of the formation of planets and planetesimals.

Sekiya (1998) was the first to find the appearance of density cusps in the above type of analysis, but he curiously dismisses the "seeming infinite density" as "due to [an] oversimplified analysis." In contrast, we believe, as already hinted above, that the appearance of density cusps, i.e., the phenomenon of "gravitational precipitation" is intimately tied to the process of GI, and is indeed crucial to understanding how the formation of planetesimals might occur in nature.

\subsection{Saturating the Particulate Layer - Qualitative Understanding}

The cusps which appear in constant $R i$ particle density profiles become singular, i.e. reach infinite midplane volume-densities, for finite particulate surface-densities. Critical profiles cannot be constructed for higher values of $\Sigma_{\mathrm{p}}$. We argue that the particulate layer 
becomes "saturated" at this critical surface density and excess solids will precipitate to the midplane and undergo GI.

The value of this critical surface density is found by taking the $\rho_{\mathrm{p}}(0) \rightarrow \infty$ limit of the general result for the surface density as a function of midplane density, equation (22) in Sekiya (1998):

$$
\Sigma_{\mathrm{p}, \mathrm{c}}=2 \sqrt{R i_{\mathrm{c}}} \eta r \rho_{g} \cdot s(\psi)
$$

where

$$
s(\psi) \equiv(1+\psi) \ln [(1+\psi+\sqrt{1+2 \psi}) / \psi]-\sqrt{1+2 \psi},
$$

is an order unity term which depends only on the (typically weak) self-gravity of the gas: $\psi \equiv 4 \pi G \rho_{\mathrm{g}} / \Omega_{\mathrm{K}}^{2} \approx 1.9 / Q_{g}$. The height of this critical layer can be found similarly from equation (21) of Sekiya (1998):

$$
\begin{aligned}
H_{\mathrm{p}, \mathrm{c}} & =\sqrt{R i_{\mathrm{c}}} \eta r \cdot h(\psi), \\
h(\psi) & \equiv \sqrt{1+2 \psi}-\psi \ln [(1+\psi+\sqrt{1+2 \psi}) / \psi] .
\end{aligned}
$$

These self gravitational terms can be approximated over a wide range of $Q_{\mathrm{g}}$ values by: $s\left(Q_{\mathrm{g}}\right) \approx 1.8\left(Q_{\mathrm{g}} / 10\right)^{0.35}$ and $h\left(Q_{\mathrm{g}}\right) \approx 0.66\left(Q_{\mathrm{g}} / 10\right)^{0.18}$.

When the self-gravitational terms are neglected, we see that the particulate mass $\Sigma_{\mathrm{p}, \mathrm{c}} \approx$ $\rho_{\mathrm{g}} H_{\mathrm{p}, \mathrm{c}}$ that can be stirred by midplane shear is equal to the mass of gas in the layer. This result has a simple intuitive interpretation via a dust-storm analogy. A fierce wind-storm in the desert can pick up a lot of dust via the Kelvin-Helmholtz instabilities that ruffle the interface between the air and the desert floor. However, the wind cannot pick up the sand of the whole desert. There is a maximum amount of dust with which air can be laden before as much dust falls out of the air as is picked up by it. In the case of a desert storm, this amount depends on hard the wind is blowing. In the nebular disk, the strength of the "wind" is fixed by relative mechanical balance considerations (depending on the parameter $\eta$ ), and it should not be too surprising, given the lack of intrinsic scales in the problem (when we ignore a role for self-gravity), that this saturation level is roughly reached when the mass of dust is equal to the mass of the layer of air in which the dust is embedded.

To derive this result more quantitatively (but still roughly), we consider how the KHI depends on $\Sigma_{\mathrm{p}}$ and the particle layer thickness, $H_{\mathrm{p}}$, for low and high values of $\rho_{\mathrm{p}} / \rho_{\mathrm{g}}$. We do this by making the crude approximation $\partial \rho_{\mathrm{p}} / \partial z \sim \rho_{\mathrm{p}} / H_{\mathrm{p}} \approx \Sigma_{\mathrm{p}} / H_{\mathrm{p}}^{2}$, which allows us to express:

$$
R i \sim \frac{\left(\rho_{\mathrm{g}} H_{\mathrm{p}}+\Sigma_{\mathrm{p}}\right)^{3}}{\left(\eta r \rho_{\mathrm{g}}\right)^{2} \Sigma_{\mathrm{p}}} .
$$

When $\rho_{\mathrm{p}} \ll \rho_{\mathrm{g}}$, the Richardson number scales as $R i \propto H_{\mathrm{p}}^{3} / \Sigma_{\mathrm{p}}$. Thus, maintaining the balance between buoyancy and shear as $\Sigma_{\mathrm{p}}$ increases requires the particle layer to become 
slightly thicker (as is seen in the detailed solutions), and will lead to a density increase: $\rho_{\mathrm{p}} \sim \Sigma_{\mathrm{p}} / H_{\mathrm{p}} \propto \Sigma_{\mathrm{p}}^{2 / 3}$.

When the particle density becomes large, $\rho_{\mathrm{p}} \gg \rho_{\mathrm{g}}$, it is no longer possible to maintain KHI since $R i \propto \Sigma_{\mathrm{p}}^{2}$. While the buoyancy is relatively constant (as long as we can still ignore self-gravity), the shear actually decreases with added mass, $\partial v_{\phi} / \partial z \propto\left(\partial \rho_{\mathrm{p}} / \partial z\right) \rho^{-2} \propto \Sigma_{\mathrm{p}}^{-1}$, because the velocity contrast is diminished as more of the material rotates at speeds closer to Keplerian. From this simplified analysis one finds that the maximum $\Sigma_{\mathrm{p}}$ which can be supported by the KHI is: $\Sigma_{\mathrm{p}, \mathrm{c}} \sim \sqrt{R i_{\mathrm{c}}} \eta r \rho_{\mathrm{g}}$, in agreement with the detailed result except for the self-gravitational correction factor.

As already hinted upon, saturation does not lead to GI of the entire particle layer unless the gas disk is already self-gravitating: $Q_{\mathrm{p}} \approx \Omega^{2} H_{\mathrm{p}, \mathrm{c}} /\left(\pi G \Sigma_{\mathrm{p}, \mathrm{c}}\right) \sim Q_{\mathrm{g}}$. Thus, only the unstirred particles in excess of $\Sigma_{\mathrm{p}, \mathrm{c}}$ should undergo GI initially. Self-gravity is important largely for the $s(\psi)$ factor. If we were to take $\psi \rightarrow 0$, which can be shown to be equivalent to setting $g_{z}=\Omega^{2} z$ at the outset, then as a high density cusp develops, $\rho_{\mathrm{p}}(0) \gg \rho_{\mathrm{g}}$, one finds that $\Sigma_{\mathrm{p}} \propto \ln \left(\rho_{\mathrm{p}}(0)\right)$. Thus, an infinite density cusp no longer corresponds to a finite $\Sigma_{\mathrm{p}, \mathrm{c}}$. Ignoring self-gravity entirely would have caused us to miss the saturation effect in Sekiya's detailed solutions.

The fact that midplane shear can only stir a finite amount of solids is only relevant if the saturation point can be reached. Let us compare $\Sigma_{\mathrm{p}, \mathrm{c}}$ to the surface density available at cosmic abundances :

$$
\frac{\Sigma_{\mathrm{p}, \mathrm{c}}}{\Sigma_{\mathrm{p}, \odot}} \sim \frac{\eta r \rho_{\mathrm{g}}}{2 \Sigma_{\mathrm{p}, \odot}} \sim\left(\frac{\Sigma_{\mathrm{g}}}{\Sigma_{\mathrm{p}}}\right)_{\odot} \frac{c_{\mathrm{g}}}{3 v_{\mathrm{K}}}
$$

where we use $\Sigma_{\mathrm{g}} \simeq 2.4 \rho_{\mathrm{g}} r c_{\mathrm{g}} / v_{\mathrm{K}}$ and $\eta \simeq 1.6\left(c_{\mathrm{g}} / v_{\mathrm{K}}\right)^{2}$. Saturation requires that the thinness of the particulate layer, $H_{\mathrm{p}} / H_{\mathrm{g}} \sim c_{\mathrm{g}} / v_{\mathrm{K}} \sim 1 / 30$, equalize the space densities $(\rho)$ of particles and gas. Our estimate indicates that saturation may be possible at cosmic abundances (i.e. $\Sigma_{\mathrm{p}, \mathrm{c}}<\Sigma_{\mathrm{p}, \odot}$ in the outer solar system, where the inclusion of ices yields a gas/solid mass ratio, $\left(\Sigma_{\mathrm{g}} / \Sigma_{\mathrm{p}}\right)_{\odot} \simeq 57$. Saturation at cosmic abundances is unlikely if only rocks are present, $\left(\Sigma_{\mathrm{g}} / \Sigma_{\mathrm{p}}\right)_{\odot} \simeq 240$. To obtain more accurate answers, detailed solutions must be used to determine whether and how much enhancement is required. The simplified treatment shows the general trends that colder disks require less enhancement. It also tells us that total disk mass is relatively unimportant, until the self-gravity factor $s(\psi)$ comes into play.

\section{3. $\quad$ Required Enhancement Values}

In this section we compute more accurate values for the enhancement of solids, or the depletion of gas, required for the onset of GI in various disk models. For the reasons described 
earlier, we adopt the "saturation" threshold (required to precipitate out a midplane layer of solids with a formally infinite space density):

$$
\Sigma_{\mathrm{p}}>\Sigma_{\mathrm{p}, \mathrm{c}}
$$

as the criterion for the onset of GI. This is clearly a more conservative approach than considering the Roche stability criterion which only requires a high but finite midplane space density, equation (2). In practice, because saturation is reached so quickly once conditions become appropriate for precipitation, the two criteria are virtually identical, except for the cases when the gas is a considerable aid to GI. In such extreme conditions of massive or very cold disks, we revert to the Toomre criterion, $Q_{\mathrm{p}}<1$, to assess the possibility for GI.

A disk model which is gravitationally stable at cosmic abundances can be made unstable in several ways, as shown for the MSN in Fig. 2. Holding the gas content fixed, increasing $\Sigma_{\mathrm{p}}$ will yield GI at a value, $\Sigma_{\mathrm{p}, \mathrm{u}}$, which is typically $\Sigma_{\mathrm{p}, \mathrm{c}}$. The amount of enhancement $\mathcal{E} \equiv \Sigma_{\mathrm{p}, \mathrm{u}} / \Sigma_{\mathrm{p}, \odot}$ needed for GI is shown for various models in Fig. 3.

The inverse process, holding the particle content fixed and lowering $\Sigma_{\mathrm{g}}$ until GI occurs at a value $\Sigma_{\mathrm{g}, \mathrm{u}}$, works equally well. The depletion factors, $\mathcal{D}=\Sigma_{\mathrm{g}, \odot} / \Sigma_{\mathrm{g}, \mathrm{u}}$, defined so that $\mathcal{D} \geq 1$, are plotted in Fig. 4 .

One can think of the gas depletion or solid enhancement scenarios as reflecting the mechanism which gives rise to solid/gas ratio enhancements and the physical conditions at the time of planetesimal formation. There is no essential difference between the two procedures except that enhancing solids yields a higher surface density. The reason that required depletion factors are larger, $\mathcal{D}>\mathcal{E}$, typically by factors of $1.5-2$, is the effect of self-gravity represented by the $s(\psi)$ factor in $\Sigma_{\mathrm{p}, \mathrm{c}}$.

We find that GI requires augmenting the particle to gas ratio by factors of two to tens above cosmic, depending on the disk model and radial location. As expected, colder disks with less pressure support (and thus less vertical shear) need less enhancement. Higher mass disks also require smaller enhancement factors, but the effect is weaker. We find that GI without enhancement is possible only in the outer regions ( $>10 \mathrm{AU}$ ) of cool disks which are 10 to 15 times more massive than the MSN in these regions.

Two caveats exist in the interpretation of these results. If only rocky materials, e.g. chondrules, are enhanced and not ices, then the fractional enhancement of solids is actually $(1+W) \mathcal{E}-W$, where $W=3.2$ (or 0 ) is the cosmic ice to rock ratio outside (or inside) the iceline. Also, if planetesimal formation occurs in one of the high $\Sigma_{\mathrm{p}}$ scenarios, the total amount of solids need not exceed the MSN if the enhancement is local. 


\section{Drift Induced Enhancement}

Here we show that gas drag in a laminar disk causes global redistribution and concentration of small solids as they inspiral. It is surprising that this straightforward and robust effect has not been studied before, but the more complex case of the global evolution of solids in turbulent disks has been studied in numerical simulations (Stepinski \& Valageas 1996). In our case, the concentration of mm-sized solids occurs on $\sim 10^{6}$ year timescales, implying that chondrules could play a crucial role in triggering planetesimal formation and in the observed disappearance of dust disks around T Tauri stars.

For particles with a radius $a<9 \lambda / 4$, where $\lambda$ is the gas mean free path, gas drag follows Epstein's law (Weidenschilling 1977). For the MSN, $9 \lambda / 4 \simeq .6 \varpi^{11 / 4} \mathrm{~cm}$, so particles up to chondrule sizes can safely be treated with Epstein drag for all but the innermost regions. The drift speed due to Epstein drag in a hydrostatic gas disk is:

$$
-\frac{d r}{d t} \equiv v_{\mathrm{dr}}=2 \frac{\rho_{\mathrm{g}}}{\rho} \eta t_{\mathrm{st}} \Omega^{2} r \sim 3 \varpi^{3 / 2}\left(\frac{10 \rho_{\mathrm{s}} a}{\mathrm{~g} / \mathrm{cm}^{2}}\right) \frac{\mathrm{AU}}{10^{6} \mathrm{yrs}},
$$

where $t_{\mathrm{st}} \equiv \rho_{\mathrm{s}} a /\left(\rho_{\mathrm{g}} c_{\mathrm{g}}\right) \ll 1 / \Omega$ is the stopping time. The numerical values in $(22)$ apply to the MSN; more generally the radial dependence goes as $v_{\mathrm{dr}} \propto r^{d}$ where:

$$
d=p-q+1 / 2
$$

where we again specify $p$ as the surface density powerlaw of the gas disk: $\Sigma_{\mathrm{g}} \propto r^{-p}$. Note that $d$ depends only on gas properties, which we assume to be time constant, and not on the evolving surface density of the solids.

We will set the inertial factor $\rho_{\mathrm{g}} / \rho \approx 1$ in $(22)$ because this approximation simplifies the mathematics at no significant cost in realism. Since GI would occur (by the saturation mechanism of $\S 3.2)$ if $\rho_{\mathrm{d}}>\rho_{\mathrm{g}}$, the procedure represents a factor $\sim 2$ error at worst in non-critical circumstances. We assume that any variation in drift speeds associated with variations in $\rho_{g} / \rho$ is hidden by the larger spread that occurs when we have a spectrum of particle sizes. With this simplifying assumption, $v_{\mathrm{dr}}$ depends on the solid density and size of the particles, but not on their surface or space density.

\subsection{Evolution of a Single Particle Size}

An axisymmetric distribution of uniformly sized particles with surface density $\Sigma(r, t)$ (we drop the "p" subscript here) evolves according to the continuity equation:

$$
\frac{\partial \Sigma}{\partial t}-v_{\mathrm{dr}} \frac{\partial \Sigma}{\partial r}=\frac{\Sigma}{r} \frac{\partial}{\partial r}\left(r v_{\mathrm{dr}}\right)
$$


subject to an initial value for $\Sigma(r, 0)$. This linear, first order PDE, a Cauchy problem, can be solved by the method of characteristics to yield a general solution:

$$
\Sigma(r, t)=r^{-d-1} g\left(r_{\mathrm{i}}(r, t)\right)
$$

where

$$
r_{\mathrm{i}}(r, t)=r\left[1-(d-1) \frac{v_{\mathrm{dr}}(r) t}{r}\right]^{-\frac{1}{d-1}},
$$

is the initial location of a particle which winds up at radius $r$ at time $t$. Note that the material at a fixed position $r$ is arriving from increasingly distant locations $r_{\mathrm{i}}$ with time, regardless of the sign of $d-1 .^{2}$ The function $g(r)=r^{d+1} \Sigma(r, 0)$ is determined by the initial conditions.

Consider a surface density which initially has a power-law profile with a cutoff at some outer radius: $\Sigma(r, 0)=\Sigma_{0} r^{-n}$ if $r<r_{\mathrm{o}}$ and $\Sigma(r, 0)=0$ otherwise. The surface density evolves as:

$$
\Sigma(r, t)=\Sigma_{0} r^{-d-1} r_{\mathrm{i}}^{d+1-n}(r, t),
$$

if $r_{\mathrm{i}}(r, t)<r_{\mathrm{o}}$, i.e. if the material comes from within the disk cutoff, and $\Sigma(r, t)=0$ otherwise.

The concentration of disk material at a given location is:

$$
\mathcal{C}(r, t) \equiv \frac{\Sigma(r, t)}{\Sigma(r, 0)}=\left[\frac{r_{\mathrm{i}}(r, t)}{r}\right]^{3 / 2-q+(p-n)},
$$

where the requirement that $r_{\mathrm{i}}(r, t)<r_{\mathrm{o}}$ still holds, or else $\mathcal{C}=0$. We have expressed $d$ using (23). If the solids and gas share the same initial powerlaw distribution, then concentration depends only on the conditions that $3 / 2>q$ (nearly inevitable) and that the outer edge of the disk has not passed, i.e. $r_{\mathrm{i}}(r, t)<r_{\mathrm{o}}$. The maximum enhancement at any location takes the simple form: $\mathcal{C}_{\max }=\left(r_{\mathrm{o}} / r\right)^{3 / 2-q}$. This can reach factors of 10's or 100's for a disk with $r_{\mathrm{o}} \sim$ few $\times 100 \mathrm{AU}$, like observed T-Tauri disks. Note, however, that the largest concentration factors in the inner disk might not be reached because of planetesimal formation that occurs as the small solids are drifting in.

The time it takes to reach a certain enhancement level $\mathcal{C}<\mathcal{C}_{\max }$ is:

$$
t_{\mathcal{C}}(r)=\frac{\left[1-\mathcal{C}^{-\frac{d-1}{3(2-q)}}\right] r}{(d-1) v_{\mathrm{dr}}(r)}
$$

\footnotetext{
${ }^{2}$ For the special case $d=1$, the general solution (25) is still valid and $r_{\mathrm{i}}=r \exp \left[v_{\mathrm{dr}}(r) t / r\right]$.
} 
For mm-sized and larger particles this timescale is shorter that the typical disk lifetime of a few $10^{6}$ years. Thus, significant concentration can be expected for chondrules and refractory inclusions. Smaller particles, such as the matrix material in chondritic meteorites, would not have enough time to be concentrated by this mechanism.

The time evolution of uniformly sized $(a=1 \mathrm{~mm})$ particles with an initial surface density:

$$
\Sigma(r, 0)=\Sigma_{0} r^{-n} \exp \left[-\left(r / r_{\mathrm{o}}\right)^{2}\right]
$$

chosen to smooth the abrupt cutoff, is plotted in Figs. 5 and 6. The particles are embedded in untruncated gas disks corresponding to models $\mathrm{H}$ and Af, respectively, and $n=p$ is assumed. For larger $d$ values, which correspond to high drift speeds in the outer disk regions, concentration occurs on a shorter timescale. Also, when $d>1$ the enhancement is most prominent where $r_{\mathrm{i}}(r, t)=r_{\mathrm{o}}$, i.e. the radius $r$ to where the outer edge $r_{\mathrm{o}}$ has drifted by time $t$. Thus, the profile becomes flatter and eventually inverted, resulting in ringlike structures. However when $d<1$ the inner regions are preferentially concentrated, steepening the surface density profiles. For the case $d=1$ (not shown) the enhancement is uniform with radius (for $r$ such that $\left.r_{\mathrm{i}}(r, t)<r_{\mathrm{o}}\right)$.

To summarize, the magnitude by which particles of a given size are concentrated depends on the radial extent of the disk, since this controls the amount of material available to pile up. The timescale depends on the drift rates, which are highest for gas that is warm and low density and particles that are large and compact.

\subsection{Particle Size Distributions}

Generalizing our results to the more realistic case of a spectrum of particle sizes is straightforward since particles of a given size evolve independently at their respective drift speed if we adopt the basic premise that particle collisions do not lead to particulate growth. Initially we assume a distribution of particle sizes such that the surface density of particles with a size between $a$ and $a+d a$ is $\sigma_{0}(r) N(a) a^{3} d a$. The size distribution, initially independent of disk radius, is typically assumed to be a simple powerlaw, $N(a) \propto a^{-s}$, with upper and lower size cutoffs, $a_{\max }$ and $a_{\mathrm{min}}$. The mass distribution is tilted towards large (small) particles if $s<4(s>4)$.

Particles of a given size evolve according to (25), and integration over the size distribution yields the total surface density:

$$
\Sigma(r, t)=\int_{a_{\min }}^{a_{\max }} r^{-d-1} g\left[r_{\mathrm{i}}(r, t ; a)\right] N(a) a^{3} d a,
$$


where we must now take into account the dependence of $R_{\mathrm{i}}$ on particle size via the drift speed. Care must be taken in evaluating this integral since the upper size cutoff, $a_{\max }$, varies with $R$ and $t$, because of the requirement that material not come from beyond a certain radius. Even for the case of a disk with no sharp edge, eqn. (30), a cutoff must be placed at some large finite disk radius because, when $d>1$, material formally drifts in from infinity in a finite time. This effect is not physical since the formula for the drift speed must be modified when it exceeds the sound speed, which occurs beyond 500 AU for millimeter sized solids. An insignificant amount of material lies at such radii, and we may eliminate the mathematical problem by imposing a cutoff. Our final results are insensitive to the location of the cutoff as long as it is imposed in the exponential tail, $r>r_{0}$.

The numerical integrations were performed using a fifth order Romberg method to deal with the large derivatives present in the kernel. Figs. 7 and 8 show the time evolution of the enhancement $\Sigma(r, t) / \Sigma(r, 0)$ of particles with a distribution of sizes and masses that is characteristic of chondrules: $a_{\min }=.01 \mathrm{~mm}, a_{\max }=1 \mathrm{~mm}, s=3$. We again use (30) for

$\Sigma(r, 0)$, with $r_{\mathrm{o}}=250 \mathrm{AU}$, with the gas disks following models $\mathrm{H}$ and Af. The main effect of introducing a size distribution is to reduce and broaden the amount of concentration since particles of different sizes drift at different rates.

\subsection{Saturation Criterion}

In order to determine whether the concentration due to gas drag only is enough to yield GI according to the saturation mechanism of $\S 3$ we need to make choices about an "initial" size distribution of particles. If the particles are uniformly sized (a false assumption) then enhancement factors are much larger than required for GI. Here we make a more reasonable assumption that initially the disk contains solar abundances of matrix material with a size distribution: $0.1 \mu \mathrm{m}<a<0.1 \mathrm{~mm}$ with $s=4$, augmented by $50 \%$ (in mass) with chondrules having the size distribution as above (in $\S 4.2$ ). The time evolution of the enhancement due to these two components in model Af at $1 \mathrm{AU}$ is shown in Fig. 9. The maximum factor of 10 in enhancement is almost exactly the factor 10 that is required for marginal GI inside the iceline, see Fig. 3. The resultant 5 to 1 ratio of chondrules to matrix material is slightly higher than the 4 to 1 ratio seen in ordinary chondrites. On the other hand, much of the matrix material in chondritic meteorites may be chondrule fragments, so the empirical enhancement may be considerably higher than represented by the nominal chondrule:matrix ratio of 4:1. In such a picture, planetesimal formation is triggered by the appearance (plus recycling and concentration) of chondrules in the nebular disk, with the ratio of chondrule to pristine matrix required to trigger GI larger in the inner solar system than the outer, 
consistent with the trends running from ordinary chondrites to carbonaceous chondrutes.

For model $\mathrm{H}$ at $1 \mathrm{AU}$, concentration under the assumptions described in the previous paragraph yields enhancement by only a factor of 7 , not quite enough for GI. Note that if the introduction of chondrules (say, by the rock recycling mechanism of the $\mathrm{x}$-wind theory) occurs not all at once in the beginning, but is delayed in time or is continuous in operation, then the enhancement factors of mm-sized bodies relative to the pristine matrix and gas (which drains continuously into the central star) may become significantly larger than the simple models illustrated above.

At larger radii where ice is present, concentration factors depend on the size distribution of the icy material, a highly uncertain quantity. If the ice is not much larger than centimeter sized, then its lower internal density will cause it to concentrate on similar timescales as chondrules, and GI becomes possible.

For the somewhat arbitrary parameters we have chosen, we conclude that aerodynamic drift provides a significant amount of concentration, but not definitively enough to cause midplane GI. Thus, in the case of our own solar system, one of the other solid/gas ratio enhancing mechanisms discussed in the introduction may be necessary.

\section{Discussion}

In this paper we have shown that planetesimals can form by midplane gravitational instabilities despite Kelvin-Helmholtz stirring if the ratio of particle to gas surface densities is increased above cosmic abundances. We have also presented a simple drift mechanism which can provide most or all of the particle enhancement required for this to occur. This mechanism has the advantage that it must operate in passive protoplanetary disks, and does not depend on assumptions about accretion physics. There are some attractive features of this scenario not yet discussed.

Curiously, the mass in our planetary system appears to be significantly truncated outside of 40 AU, i.e. in the Kuiper belt region and beyond (Trujillo \& Brown 2001), whereas T-Tauri disks typically extend to several hundred AU. The size disparity is even greater if Uranus and Neptune migrated from a location interior to Saturn's orbit (Thommes, Duncan, \& Levison 1999). The process of drift induced enhancement offers an explanation. The outer disk is drained relatively of its solid resources by inward particulate drift, and thereby becomes or remains inhospitable to the formation of planetesimals.

Another troubling aspect of planet formation theories is the "Type-I" migration of 
Earth-sized and larger (but not large enough to open a gap) bodies due to density waves torques exerted on the gas disk (Ward 1997). When the resonant torques are assumed to damp locally in the disk, Earth-mass bodies (at $5 \mathrm{AU}$ ) migrate inwards in $10^{5}$ years in a MSN disk. This timescale is inversely proportional to the mass of the body, but increases sharply once the gap-opening mass, typically $10-100 M_{\oplus}$ is reached. However the drift speed is proportional to $\Sigma_{\mathrm{g}}$. Thus if planetesimals form due to depletion of gas below MSN values (or equivalently the enhancement of solids in a very low mass disk), then subsequent earth-mass cores suffer less migration. Gas depletion by one order of magnitude would significantly increase the survival odds of a nascent planetary system, and still leave enough gas for the formation of giant planet atmospheres.

To summarize, in conventional cosmogonies with unit sticking probabilities and cosmic abundances of solids and gas in a MSN (Lissauer 1993), planetesimal formation is easy, occurring on a time scale of $\sim 10^{4}$ yr or less, while giant planet formation is hard, requiring time scales in excess of the typical lifetimes of T Tauri disks, $\sim 3 \times 10^{6}$ yr. Overall surface density enhancements (gas and dust) above MSN values can speed up post-planetesimal growth (Thommes, Duncan, \& Levison 2002), but this solution would exacerbate the problem of the Type-I migration of planetary embryos (Ward 1997). It would also require a finely tuned mechanism to remove the considerably greater amount of extra gas and solids from the solar system.

In an unconventional cosmogony, where sticking probabilities are zero (except for the special mechanisms that produce chondrules and refractory inclusions near the protosun), where gas is depleted, and solids are enhanced relative to standard MSN values, the conditions for the formation of planetesimals and giant planets might be intimately tied to the gas-dust evolution of the nebular disks of $\mathrm{T}$ Tauri stars. The two processes would then naturally acquire similar time scales, related by a single continuous process of gravitational growth in a gas-dust disk. Attractive byproducts of this unconventional approach would be a corresponding alleviation of the problems of Type I migration and gas-disk dispersal, as well as a possible understanding of why the Kuiper belt marks a sudden apparent truncation of the primitive solar system.

A. Y. would like to thank Andrew Cumming, Jeff Cuzzi, Greg Laughlin, Geoff Marcy, and Jonathan Swift for helpful discussions and the referee for suggesting the following appendix and other improvements. Financial support was provided by a grant from the NASA Origins of Solar Systems Program. A. Y. acknowledges support from an NSF Graduate Fellowship.

\section{APPENDIX}




\section{A. Collisional Effects}

In most of this paper we have ignored the effects of collisions. Here we argue that this approach is is dynamically justified. The time between collisions is:

$$
t_{\mathrm{coll}} \simeq \frac{\rho_{\mathrm{s}} a H_{\mathrm{p}}}{\Sigma_{\mathrm{p}} c_{\mathrm{p}}} \approx \frac{\rho_{\mathrm{s}} a}{\Sigma_{\mathrm{p}} \Omega},
$$

which agrees within factors of order unity with the more exact results presented in Wetherill \& Stewart (1993) if we use $c_{\mathrm{p}} / H_{\mathrm{p}} \approx \Omega$ in the second equality. Thus the collisional timescale is longer than the stopping time, $t_{\text {coll }} / t_{\mathrm{st}} \approx \Sigma_{\mathrm{g}} / \Sigma_{\mathrm{p}}$ by at least an order of magnitude.

Even if collisional dynamics were able to introduce an effective viscosity, $\nu_{\text {coll }} \simeq c_{\mathrm{p}}^{2} t_{\mathrm{c}}$ (Goldreich \& Tremaine 1978), the characteristic diffusion velocity, $v_{\text {coll }} \simeq \nu_{\text {coll }} / r$ is less than the drift velocity due to Epstein drag:

$$
\frac{v_{\text {coll }}}{v_{\mathrm{dr}}} \simeq \eta \frac{\Sigma_{\mathrm{g}}}{\Sigma_{\mathrm{p}}} \lesssim 10^{-2} .
$$

If collisions could change the size distribution of solids due to fragmentation or mergers then our results for drift induced enhancement ( $(4)$ would have to be modified by including

a coagulation equation, e.g. Kenyon \& Bromley (2002). However we already argued in the introduction against the effectiveness of collisional agglomeration. As for fragmentation, the presence of significant numbers of intact chondrules (the species most important for the enhancement) in meteorites shows that many were able to survive their collisional history in the nebular disk without much shattering.

\section{REFERENCES}

Blum, J. \& Muench, M. 1993, Icarus, 106, 151

Blum, J. \& Wurm, G. 2000, Icarus, 143, 138

Boss, A. P. 2000, ApJ, 536, L101

Chiang, E. I. \& Goldreich, P. 1997, ApJ, 490, 368

Cuzzi, J. N., Dobrovolskis, A. R., \& Champney, J. M. 1993, Icarus, 106, 102

Cuzzi, J. N., Hogan, R. C., Paque, J. M., \& Dobrovolskis, A. R. 2001, ApJ, 546, 496

de la Fuente Marcos, C. \& Barge, P. 2001, MNRAS, 323, 601 
Fessler, J. R., Kulick, J. D., \& Eaton, J. K. 1994, Physics of Fluids, 6, 3742

Gammie, C. F. 1996, ApJ, 457, 355

Gilliland, R. L. et al. 2000, American Astronomical Society Meeting, 196, 0202.

Goldreich, P. \& Lynden-Bell, D. 1965, MNRAS, 130, 97

Goldreich, P. \& Tremaine, S. D. 1978, Icarus, 34, 227

Goldreich, P. \& Ward, W. R. 1973, ApJ, 183, 1051

Goodman, J. \& Pindor, B. 2000, Icarus, 148, 537

Gounelle, M., Shu, F. H., Shang, H., Glassgold, A. E., Rehm, K. E., \& Lee, T. 2001, ApJ, 548, 1051.

Haisch, K. E., Lada, E. A., \& Lada, C. J. 2001, ApJ, 553, L153.

Hayashi, C. 1981, Progress of Theoretical Physics Supplement, 70, 35

Hollenbach, D. J., Yorke, H. W., \& Johnstone, D. 2000, Protostars and Planets IV (Book Tucson: University of Arizona Press; eds Mannings, V., Boss, A. P., Russell, S. S.), p. 401

Jones, A. P., Tielens, A. G. G. M., \& Hollenbach, D. J. 1996, ApJ, 469, 740

Kenyon, S. J. \& Bromley, B. C. 2002, AJ, 123, 1757

Laughlin, G. 2000, ApJ, 545, 1064.

Lissauer, J. J. 1993, ARA\&A, 31, 129

Marcy, G. W. \& Butler, R. P. 1998, ARA\&A, 36, 57.

Marshall, J. \& Cuzzi, J. 2001, Lunar and Planetary Institute Conference, 32, 1262

Mayor, M. \& Queloz, D. 1995, Nature, 378, 355.

Nakagawa, Y., Sekiya, M., \& Hayashi, C. 1986, Icarus, 67, 375

Osterloh, M. \& Beckwith, S. V. W. 1995, ApJ, 439, 288

Rubin, A. E. 2000, Earth Science Reviews, 50, 3. 
Safronov, V. S. 1969, Evolution of the Protoplanetary Cloud and Formation of the Earth and the Planets. (Moscow: Nauka Press), [NASA Tech. Trans. F-677]

Sekiya, M. 1983, Progress in Theoretical Physics, 69, 1116

Sekiya, M. 1998, Icarus, 133, 298

Shu, F. H. 1984, IAU Colloq. 75: Planetary Rings, 513.

Shu, F. H., Johnstone, D., \& Hollenbach, D. 1993, Icarus, 106, 92

Shu, F. H., Shang, H., \& Lee, T. 1996, Science, 271, 1545

Shu, F. H., Shang, H., Gounelle, M., Glassgold, A. E., \& Lee, T. 2001, ApJ, 548, 1029.

Squires, K. D. \& Eaton, J. K. 1991, Physics of Fluids, 3, 1169

Stepinski, T. F. \& Valageas, P. 1996, A\&A, 309, 301

Supulver, K. D., Bridges, F. G., Tiscareno, S., Lievore, J., \& Lin, D. N. C. 1997, Icarus, 129, 539

Thi, F. W. \& et al. 2000, IAU Symposium, 202, E84

Thommes, E. W., Duncan, M. J., \& Levison, H. F. 1999, Nature, 402, 635

Thommes, E. W., Duncan, M. J., \& Levison, H. F. 2002, Icarus, in press

Trujillo, C. A. \& Brown, M. E. 2001, ApJ, 554, L95.

Ward, W. R. 1976, Frontiers of Astrophysics (Cambridge, MA: Harvard Univ. Press; ed Avrett, E. H.), p. 1

Ward, W. R. 1997, ApJ, 482, L211

Weidenschilling, S. J. 1995, Icarus, 116, 433

Weidenschilling, S. J. 1977, MNRAS, 180, 57

Weidenschilling, S. J. \& Cuzzi, J. N. 1993, Protostars and Planets III, 1031

Wetherill, G. W. \& Stewart, G. R. 1993, Icarus, 106, 190

Youdin, A. N. \& Shu, F. H. 2002, in prep.

Zebker, H. A., Marouf, E. A., \& Tyler, G. L. 1985, Icarus, 64, 531. 
This preprint was prepared with the AAS IATEX macros v5.0. 


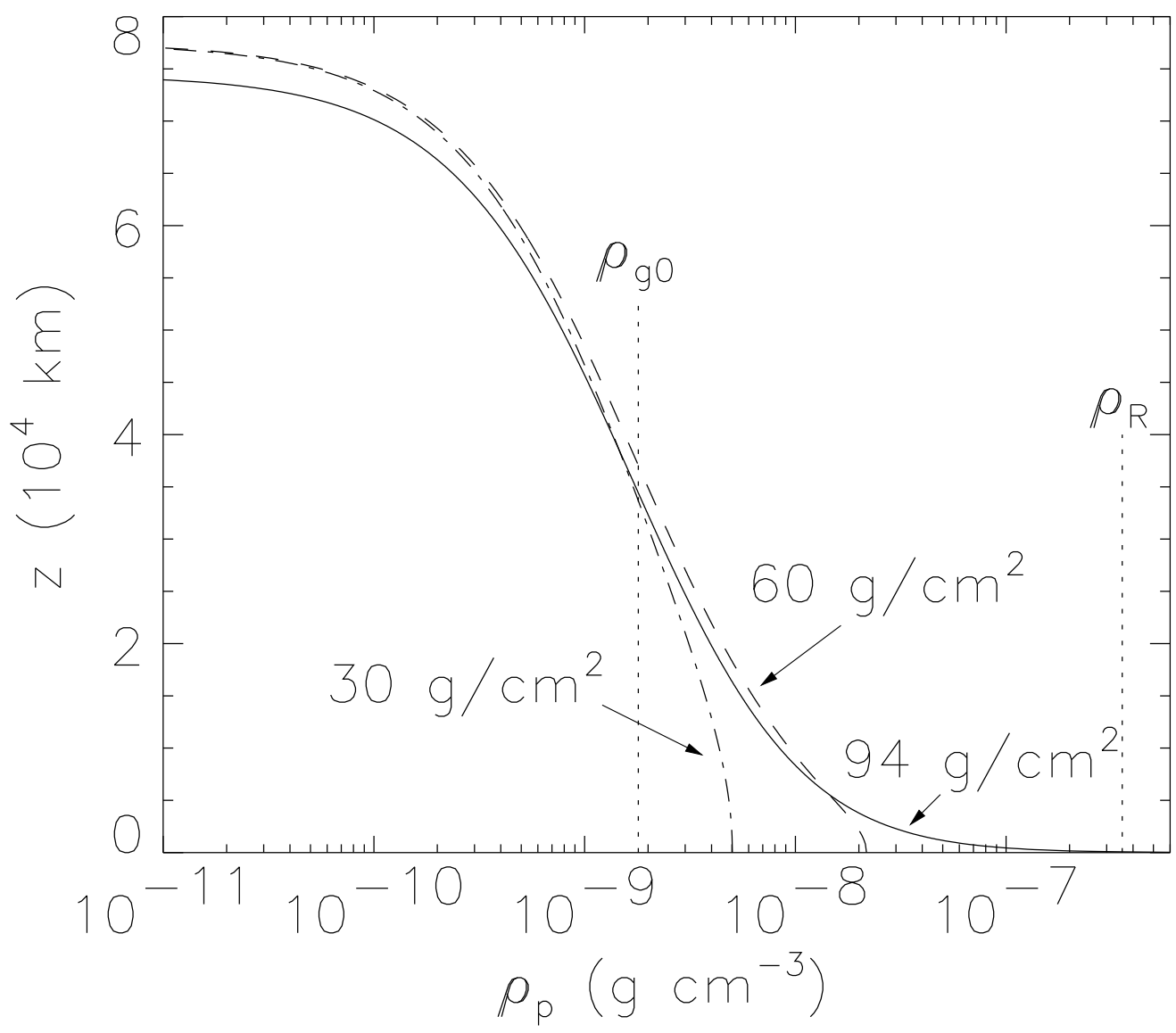

Fig. 1.- Vertical profiles of particle density at $1 \mathrm{AU}$, for small solids stirred by the KelvinHelmholtz instability. The gas is described by model A and held fixed. As $\Sigma_{\mathrm{p}}$ increases from $30 \mathrm{~g} / \mathrm{cm}^{2}$, the value at solar abundances (with ice), a midplane density cusp develops, becoming infinite at $\Sigma_{\mathrm{p}}=\Sigma_{\mathrm{p}, \mathrm{c}}=94 \mathrm{~g} / \mathrm{cm}^{2}$ for this model. Also shown are the gas, $\rho_{g}$, and the Roche, $\rho_{R}$, densities. 


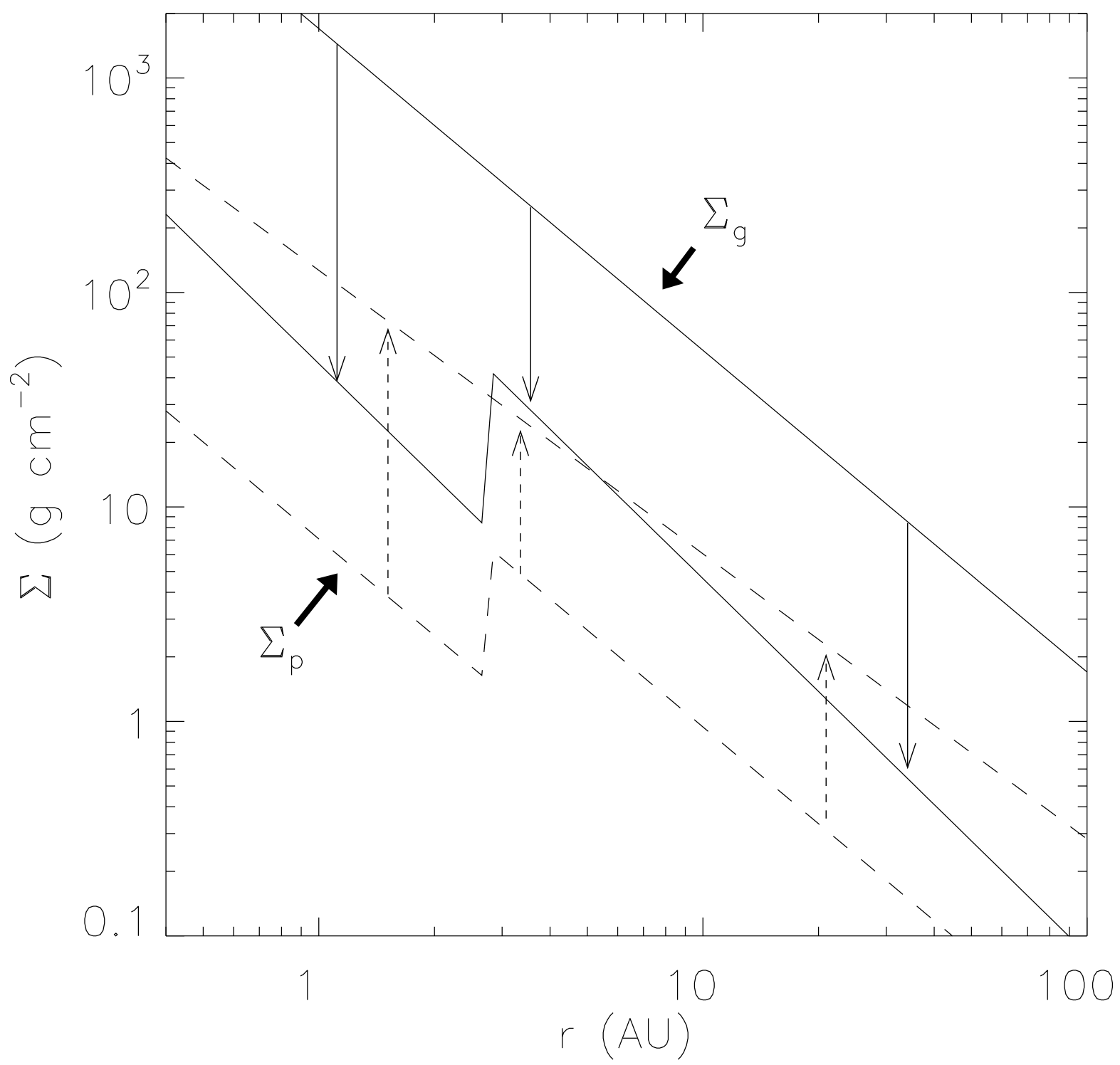

Fig. 2.- The MSN disk model can be made gravitationally unstable by either enhancing the solids (increasing $\Sigma_{\mathrm{p}}$ as indicated by the dashed arrows), by depleting the gas (decreasing $\Sigma_{\mathrm{g}}$ as indicated by the solid arrows), or by some combination of the two (not shown). Note that instability is local, and need not occur simultaneously at different radii. 


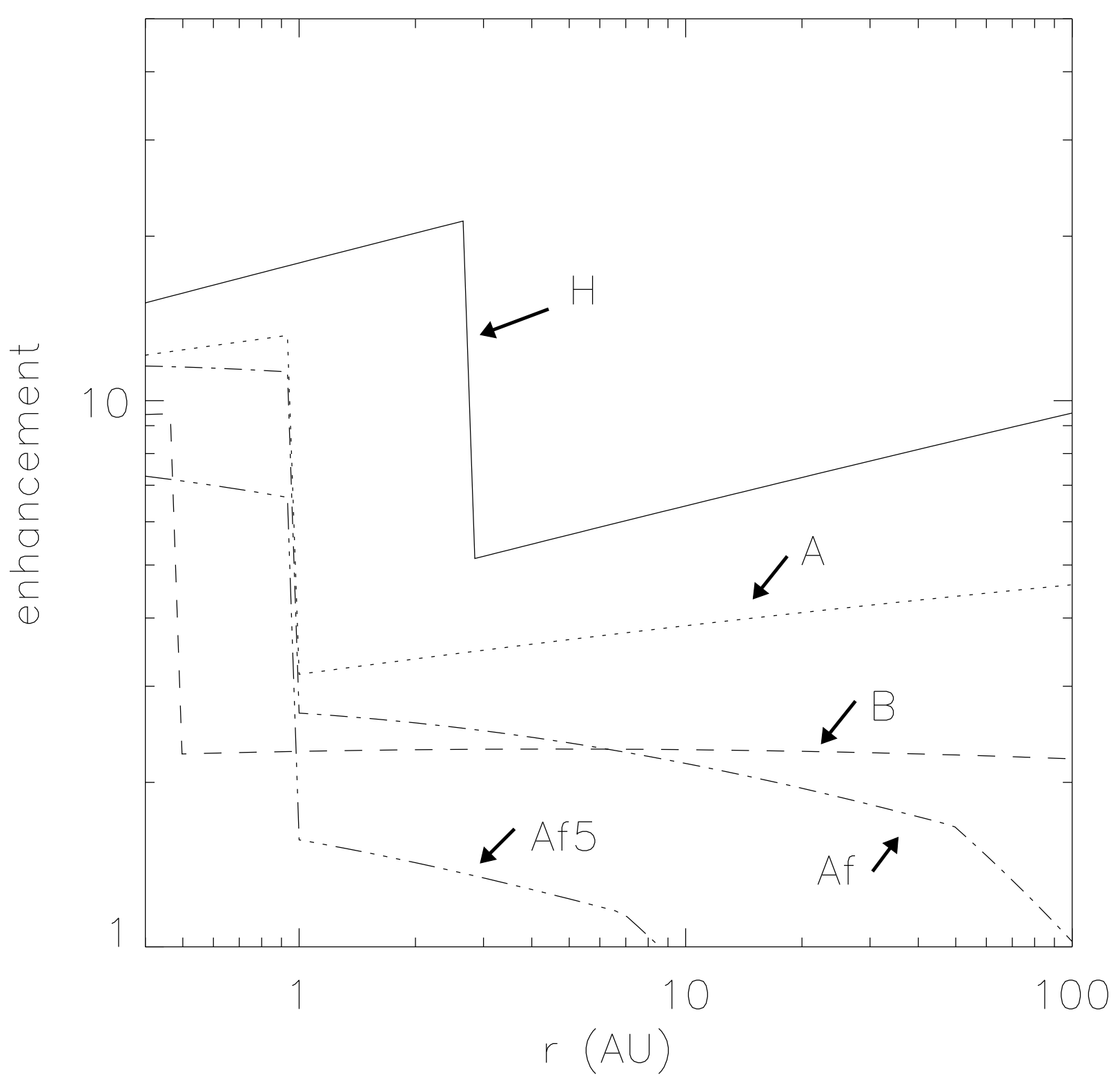

Fig. 3.- Enhancement factor, $\mathcal{E}$, required for GI vs. radius for various models. The discontinuity occurs because larger enhancements are needed inside the iceline. Kinks in higher mass models (Af,Af5) occur when $Q_{\mathrm{p}}<1$ occurs before saturation. 


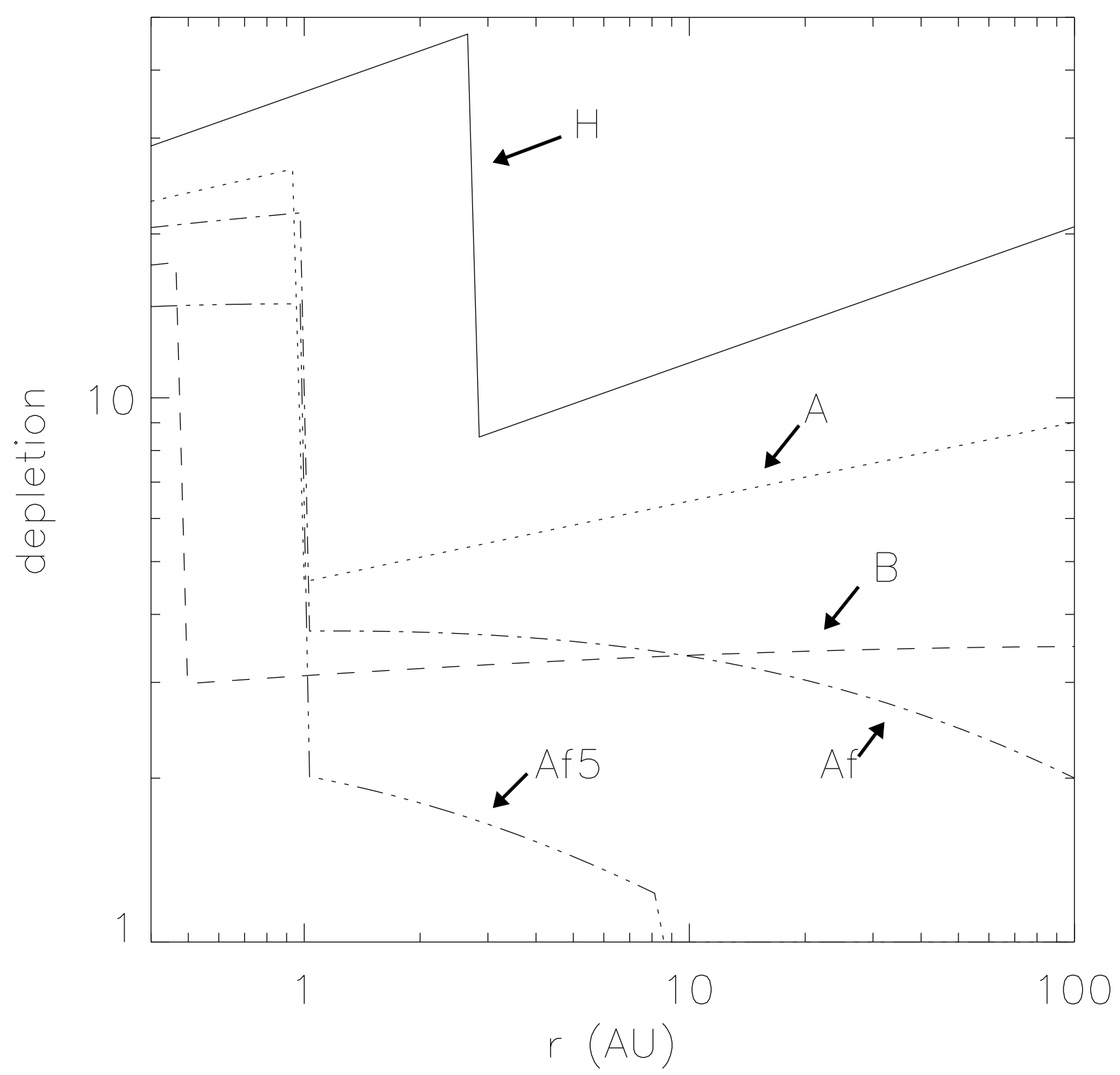

Fig. 4.- Depletion factor, $\mathcal{D}$, required for GI vs. radius. 


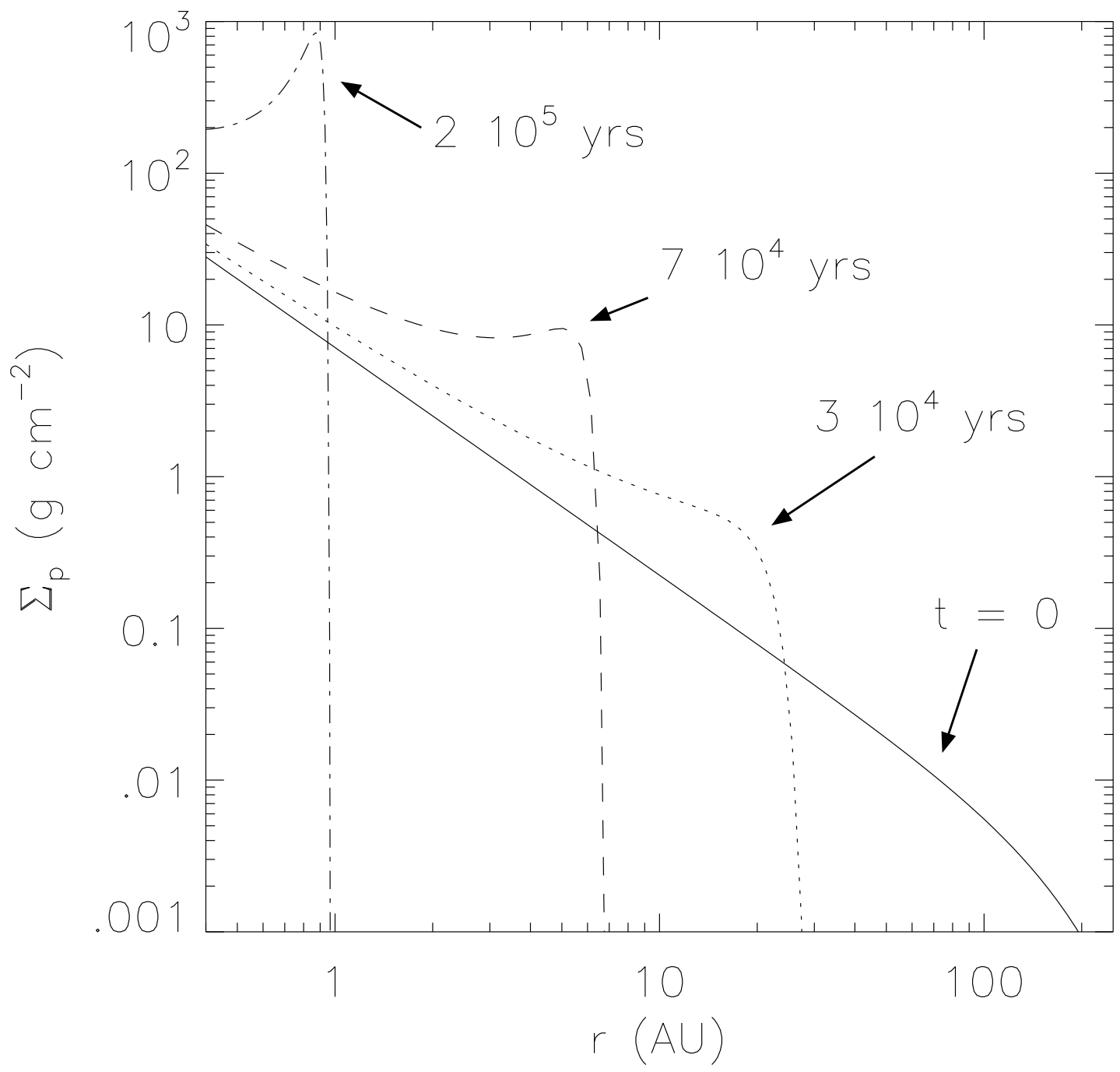

Fig. 5. - Time evolution of the surface density of mm-sized particles with solid densities, $\rho_{\mathrm{s}}=3 \mathrm{~g} / \mathrm{cm}^{3}$, due to Epstein drag. The gas is assumed fixed at MSN values. Since $d=3 / 2>1$, the $\Sigma_{\mathrm{p}}$ profiles, initially described by eq.(30) with $R_{\mathrm{o}}=200 \mathrm{AU}$, become flatter and eventually inverted into ringlike structures. 


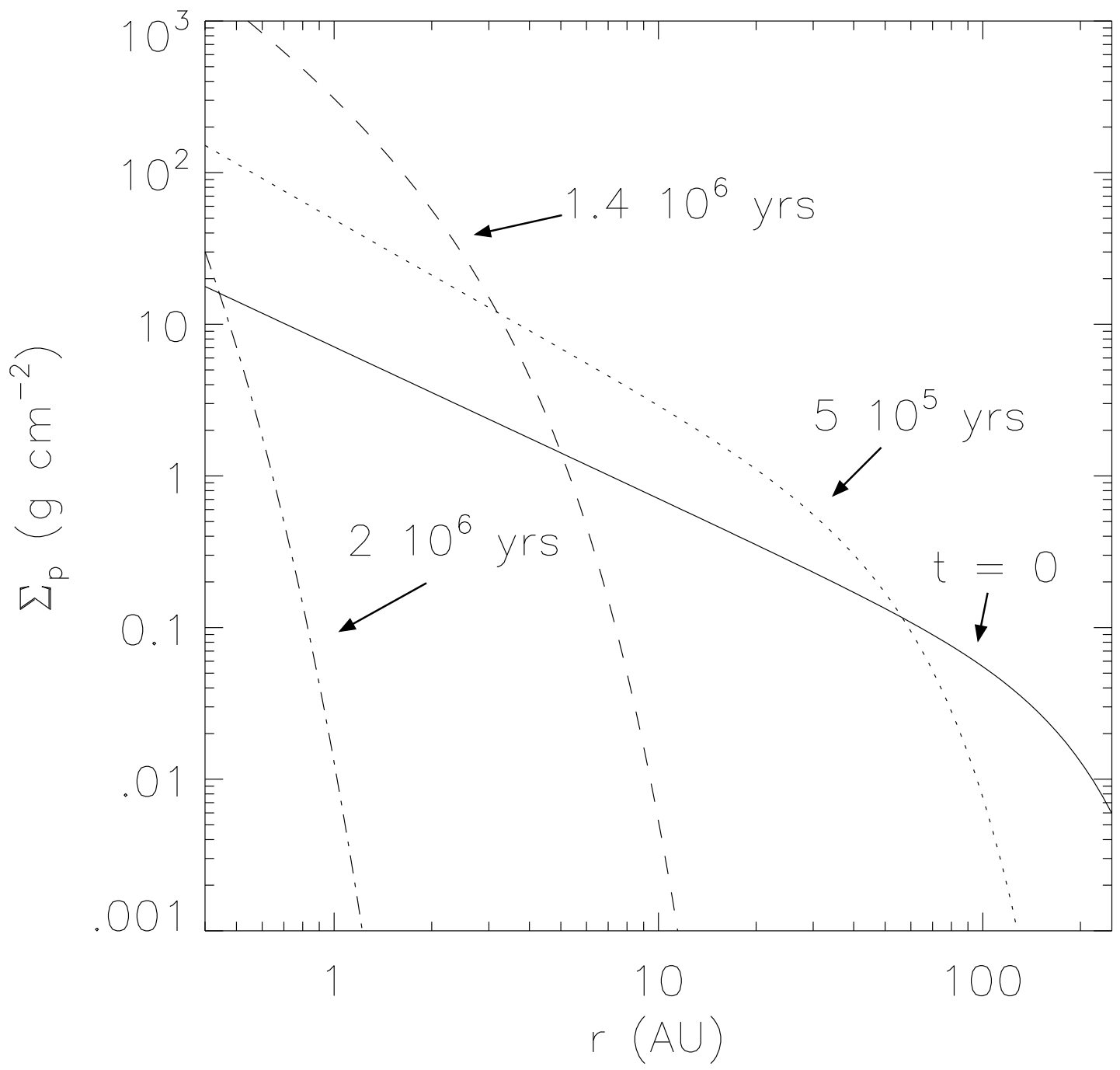

Fig. 6. - Same as Fig. 5 except the gas is described by model Af. Since $d=.87<1, \Sigma_{\mathrm{p}}$ evolves to steeper profiles. 


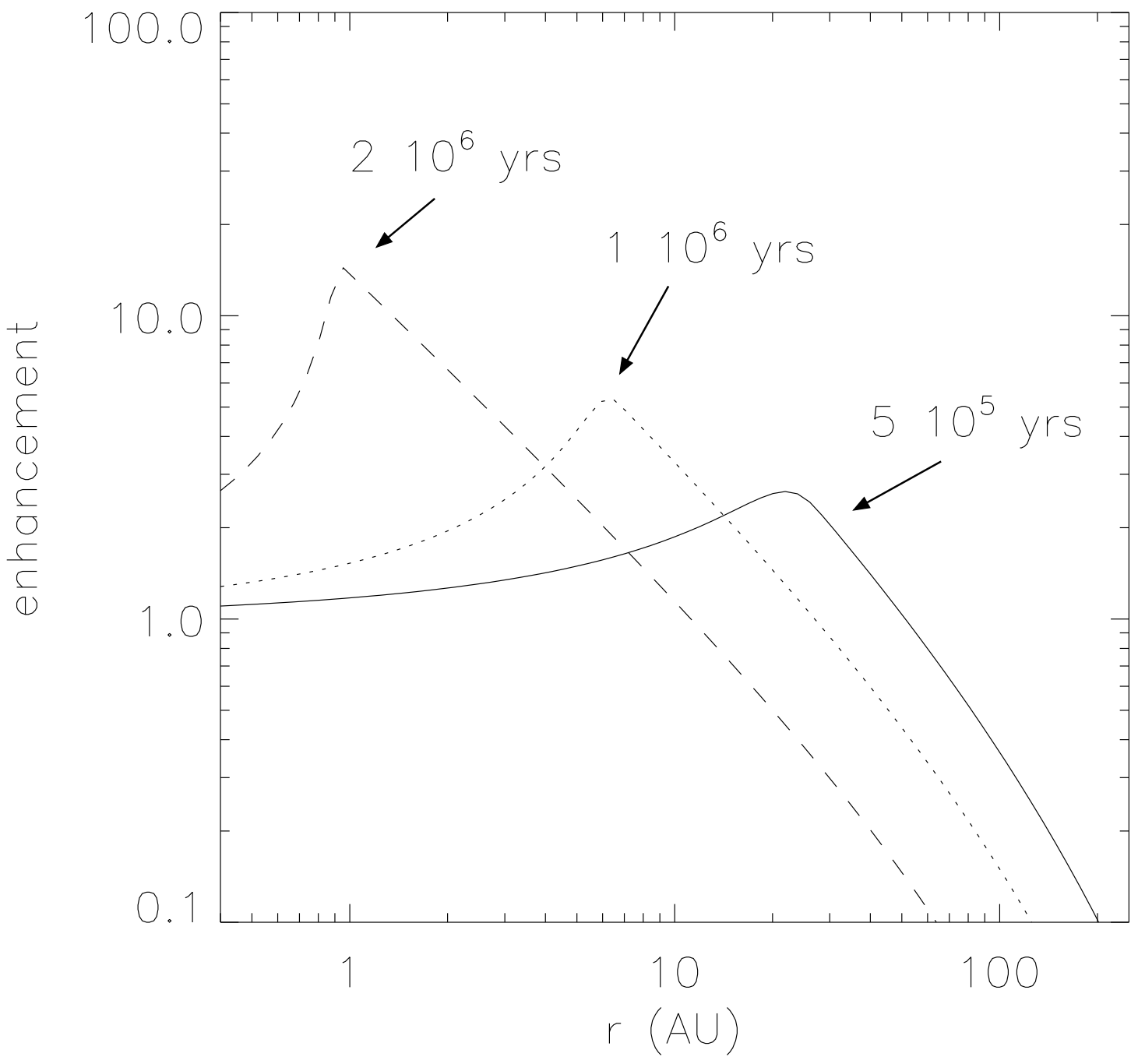

Fig. 7.- Time evolution of the surface density enhancement of solid particles with a size distribution characteristic of chondrules. The gas disk is described by the MSN. See text for details. 


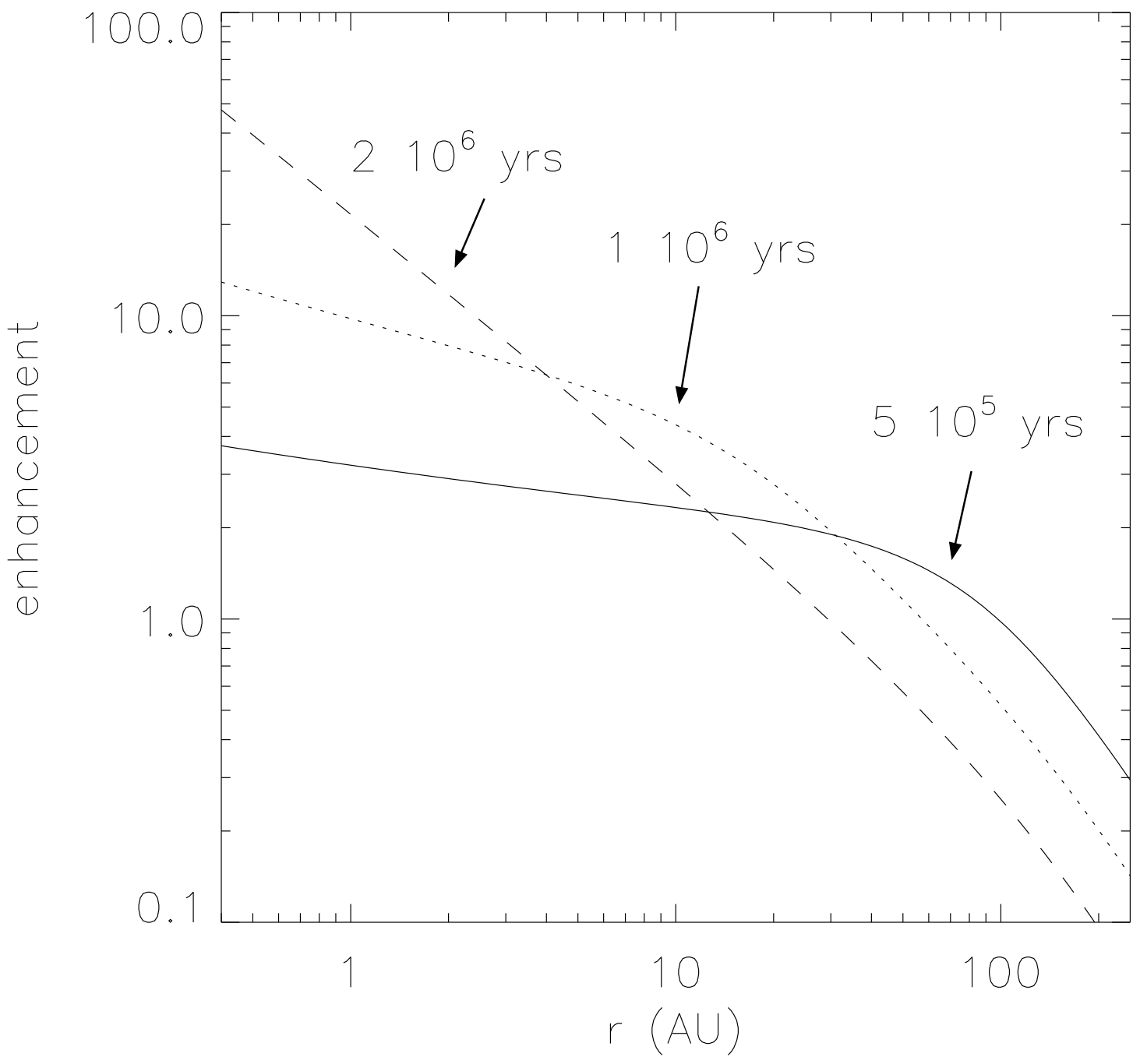

Fig. 8.- Same as Fig. 7 except the gas is described by model Af. 


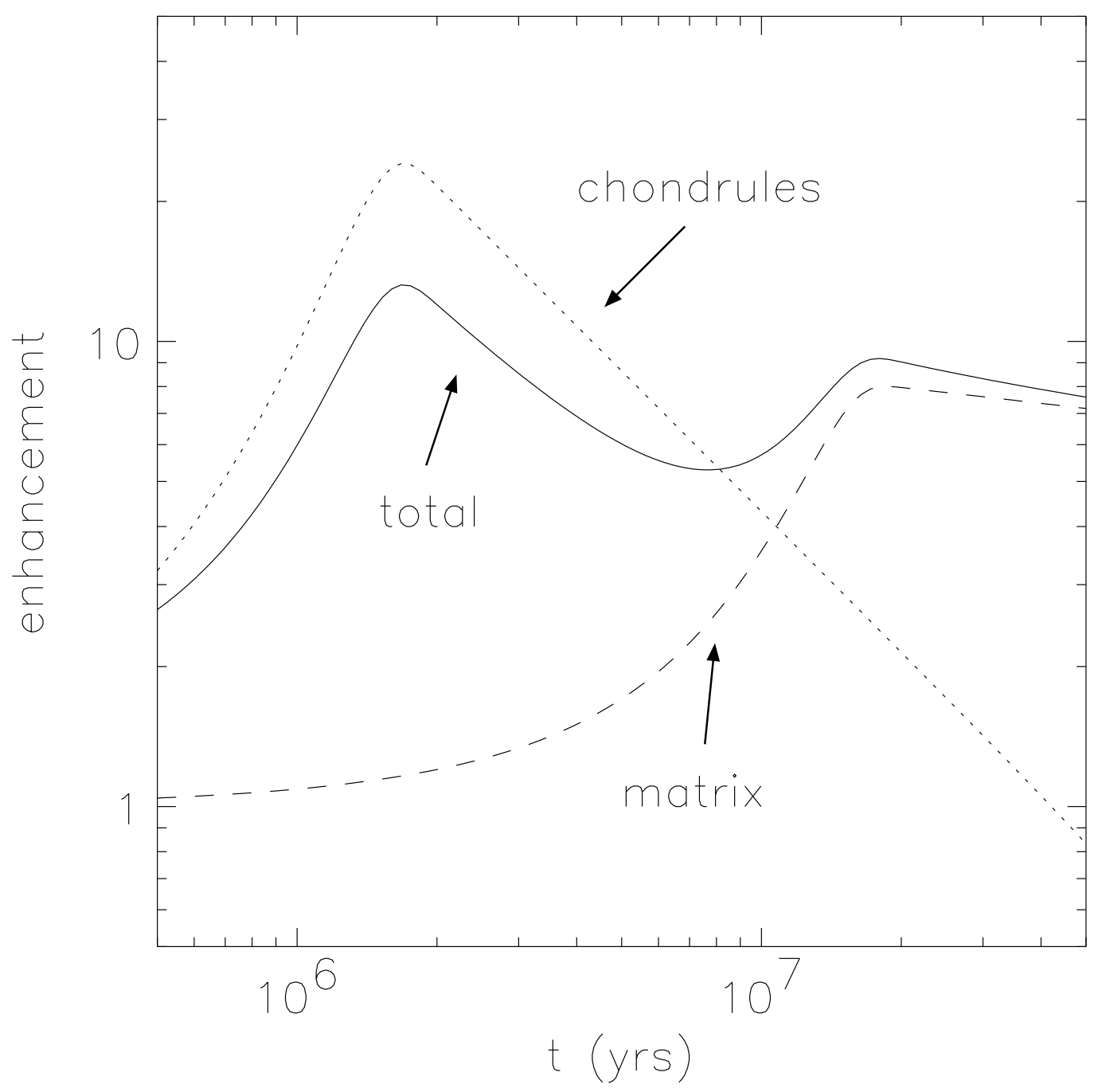

Fig. 9. - Enhancement of solids vs. time at $1 \mathrm{AU}$ in model Af for a particle disk that is $1 / 3$ chondrules and $2 / 3$ matrix material by mass. 
Table 1. Model Parameters

\begin{tabular}{ccccccc}
\hline \hline Model & $p$ & $f^{\mathrm{a}}$ & $q$ & $T_{1}(\mathrm{~K})$ & iceline $(\mathrm{AU})$ & $d^{\mathrm{b}}$ \\
\hline $\mathrm{H}$ & $3 / 2$ & 1 & $1 / 2$ & 280 & 2.7 & 1.5 \\
$\mathrm{~A}$ & $3 / 2$ & 1 & .63 & 170 & 1 & 1.37 \\
$\mathrm{~B}$ & $3 / 2$ & 1 & $3 / 4$ & 100 & .5 & 1.25 \\
$\mathrm{Af}$ & 1 & 1 & .63 & 170 & 1 & .87 \\
Af5 & 1 & 5 & .63 & 170 & 1 & .87 \\
\hline
\end{tabular}

${ }^{\mathrm{a}} f=f_{\mathrm{g}}=f_{\mathrm{i}}=f_{\mathrm{r}}$ for solar abundances.

bSee $\S 4$, eq. (23). 